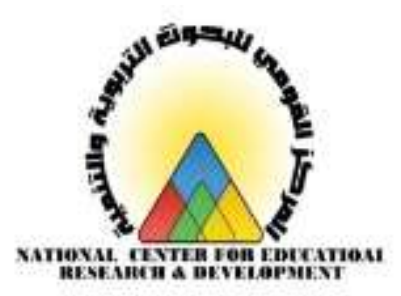

\title{
Die Sprachdiagnostik zur Förderung des Deutschlernens bei den Schülern an der Oberschule (Eine Fallstudie)
}

Bearbeitet von

\section{Reham Badr El-Din Abd El- Aziz}

Assistenzforscherin am "Nationalen Zentrum für Bildungsforschung und Entwicklung"

\section{Prof. Dr. Amal Abdallah}

Professorin an der Curricula- und Methodikabteilung

Pädagogische Fakultät

Ain Shams Universität

Prof. Dr. Duaa Abd El Karim

Professorin an der Curricula- und Methodikabteilung

Pädagogische Fakultät

Heluan Universität

Dr. Heba Kenawi

Assistenzprofessorin an der Curricula- und Methodikabteilung

Pädagogische Fakultät

Ain Shams Universität 
Die Sprachdiagnostik zur Förderung des Deutschlernens

bei den Schülern an der Oberschule (Eine Fallstudie)

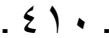

البـحث التتريوي 


$$
\text { المركن الثومي للبحوث التبويةوالنمية }
$$

التشخيص اللغهي لتمهزيز تمام الطلاب للانة

الألانية في المودلة الثانوبة (دراسة هالة)

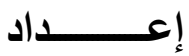

ريهام بدر الدين عبدالعزيز

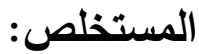

هدف البحث الى تعزيز تعلم اللغة الألمانية لطلاب المرحلة الثانوية،

واستخدام النتخيص اللغوي كأحد أساليب تقويم الأداء اللغوي للطلاب، وقياس فاعلية التتخيص اللغوي لتعزيز تعلم اللغة الألمانية لطلاب المرحلة الثانوية، وذلك من خلا استخدام التتخيص اللغوي كأداة للتقويم المصاحب لتعلم اللغة الألمانية. وقد تكونت عينة البحث من ستة طلاب ثم اختيارهم طبقًا لنتائج اختبار تشخيصي في بداية البرنامج وانقست عينة البحث (ستة طلاب) إلي ثلاثة طلاب هم الأكثر تفوقًا والثثلاثة الأخرين هم الأقل في مستوى الأداء وفقًا . §). البحث التربوي 
Die Sprachdiagnostik zur Förderung des Deutschlernens bei den Schülern an der Oberschule (Eine Fallstudie)

لنتائج التطبيق الأول للاختبار التشخيصي على جميع الطلاب، واستغرق التطبيق سبعة أسابيع خلال الفصل الدراسي الثاني من العام الدراسي ^1 ـ ب19 1 ب باجمالي مب حصة دراسية. وقد توصلت الباحثة الى النتائج التالية: وجود فروق ذات دلالة احصائية بين منوسطي درجات الطلاب في تطبيقي الاختبار التشخيصي لصالح التطبيق البعدي وذلك من خلا التحليل الكمي لنتائج اختبار ويلكوكسون، أما التحليل الكيفي فقد أظهر وجود نمو ملحوظ في أداء الطلاب في شتى المهارات اللغوية، وكذلك المهارات التواصلية باستخدام اللغة. كما نوصلت الى أن حجم تأثير التشخيص اللغوي على تعلم الطلاب للغة الألمانية يعد تأثيرًا كبيرًا جدًا.

الكلمات المفتاحية: النتخيص اللغوي، النمو اللغوي، دراسة الحالة. 


$$
\text { المركن القومي للبحوث التبوبةوالتنمية }
$$

\section{Die Sprachdiagnostik zur Förderung des Deutschlernens bei den Schülern an der Oberschule (Eine Fallstudie)}

Bearbeitet von

Reham Badr El-Din Abd El- Aziz

Assistenzforscherin am "Nationalen Zentrum für Bildungsforschung und Entwicklung"

\section{Einleitung}

Die effektive Evaluation ist ein guter Indikator für die Güte der Bildung. Der Fortschritt der heutigen Nationen wird am Qualitätsniveau des Bewertungssystems gemessen. Das Evaluationsziel wird nicht mehr die Einordnung oder Klassifizierung der Schüler, sondern die Vertiefung ihrer Lernmotivation und ihres Lerngefühls. Dies führt zu einer Erleichterung des Verständnisses. So wird die auf Evaluation basierende Bildung zum Tor zur Entwicklung des gesamten Bildungssystems. Für lange Zeit wird die Evaluation zum Synonym für die Prüfung, um endgültige Entscheidungen über die Schülerstufe zu treffen, entweder erfolgreich oder erfolglos, sodass alle . $\Sigma 1 \%$. 
Die Sprachdiagnostik zur Förderung des Deutschlernens

bei den Schülern an der Oberschule (Eine Fallstudie)

pädagogischen Anstrengungen und Aktivitäten für den Test verfügbar sind, um eine möglichst hohe Punktzahl zu erzielen (vgl. Müller 2019: 69; Ewringmann et al. 2004: 3ff.).

Gnahs und Quilling identifizierten wichtige Evaluationszwecke, nämlich Qualitätsentwicklung, Planung, Lenkung, Prüfung, Effizienzsteigerung und Kontrolle (vgl. Gnahs/ Quilling 2019: 100).

Traditionelle Evaluationsverfahren befassen sich mit der Bewertung der kognitiven Leistung der Schüler, vernachlässigen jedoch die emotionalen Seiten und Hintergründe, die sich auf die menschliche Leistung auswirken. Sie basieren eher auf einer Form der statischen Diagnostik, die darauf abzielt, den tatsächlichen Zustand einer Person $\mathrm{zu}$ erfassen. Sie zielen damit auf die Voraussage und Auswahl der erfolgreichen Schüler für bestimmte Chancen. Hingegen stehen im Mittelpunkt der pädagogischen Diagnostik die Ressourcen und Stärken der individuellen Schüler. Durch den Einsatz der 


$$
\text { المركن القومي للبحوث التبوبةوالتنمية }
$$

pädagogischen Diagnostik können Lehrer die Lernvoraussetzungen der Schüler ermitteln, ihre Lernprozesse analysieren und gezielte Fördermaßnahmen entwickeln. Wichtig dabei ist die begleitende Überprüfung der Effizienz dieser Maßnahmen. Von den neuen Evaluationsansätzen ist die dynamische Pädagogik, die Lehren, Lernen und Beurteilung in einer einzigen Einheit vereint. Diese dynamische Pädagogik ist eine Unterrichtsform, die Beurteilung, Lehrplan und Unterricht in den Dienst des Lernens stellt (vgl. Gordon/ Rajagopalan 2016: 6ff.).

Die Diagnostik gehört seit langem zur Sonderpädagogik. Durch eine frühe Diagnose kann der Lehrer seine Bemühungen auf die Bedürfnisse jedes Schülers und seinen Plan auf das Qualifikationsniveau der gesamten Klasse abstimmen. Die Diagnose bietet dem Lehrer die Möglichkeit, eine detaillierte Analyse und Berichte von Schülerreaktionen auf Aufgaben zu erstellen. Die Diagnose soll ein kontinuierliches Merkmal des Lernens sein. Sie ermöglicht es den Lehrern, die mit 
Die Sprachdiagnostik zur Förderung des Deutschlernens

bei den Schülern an der Oberschule (Eine Fallstudie)

traditionellen Tests verbundenen Ängste der Schüler zu vermeiden. Die Ergebnisse können jedoch nicht für Evaluierungszwecke wie die Bewertung oder Auswahl von Schülern für bestimmte Gelegenheiten verwendet werden (vgl. Reed 2006: 1ff.).

Die Beiträge von (Wiprächtiger-Geppert 2011) und (Reich 2006) erkannten die Sprachdiagnostik in der Sprachwissenschaft an. Bezüglich der Sprache kann die diagnostische Beurteilung als ein wichtiger Bereich angesehen werden, der sich nicht wie die Kompetenz- und Leistungstests entwickelt. Der Arbeit mit einem sprachstandsdiagnostischen Verfahren geht oftmals die Frage voraus, warum eine Sprachstandserhebung eigentlich durchgeführt wird. Individuelle Fördermaßnahmen können nur in Betracht gezogen und festgelegt werden, wenn es klar ist, inwieweit ein Schüler sich eine Fremdsprache aneignet (vgl. Geist 2014: 38f; Reed 2006: 1; Bakk. phil. 2014: 11).

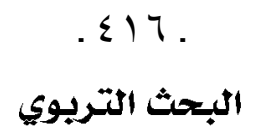




$$
\text { المركن القومي للبحوث التبوبةوالتنمية }
$$

Der Begriff Sprachdiagnostik bezieht sich auf die Untersuchung des Sprachniveaus, die Sammlung von Informationen über die Sprachbiographie des Schülers, auf die Auswertung, die Datenanalyse, die Ermittlung des Sprachentwicklungsbedarfs und auf die individuellen Entwicklungsziele. Sprachdiagnostik kann als Ausgangspunkt für die Sprachentwicklung angesehen werden. Mithilfe der Sprachdiagnose können Lehrer für jeden Schüler ein Sprachprofil erstellen. Dieses Sprachprofil wird als nützliches Instrument zur Dokumentation von formellen und informellen Sprachlernprozessen sowie von Lernfortschritten von Schülern und Bildungszielaufbau angesehen (vgl. Lengyel 2012: 8ff.).

Die Ziele der Sprachdiagnostik sind die Ermittlung vom Bedarf an Sprachförderung, die genaue Beschreibung des Sprachniveaus, der Sprachschwächen und -fähigkeiten, die Beobachtung der Sprachleistung im realen Kontext, die Bewertung des Erfolgs oder Misserfolgs der pädagogischen Maßnahmen sowie die Individualisierung der Planung des 
Die Sprachdiagnostik zur Förderung des Deutschlernens

bei den Schülern an der Oberschule (Eine Fallstudie)

Sprachunterrichts und der Sprachförderung. Eine differenzierte Bildung hilft den Lehrern, den Bewertungsprozess $\mathrm{zu}$ verstehen und so anzuwenden, wie er sein sollte. Die Lehrer sollen unterschiedliche Bewertungsmethoden anwenden, die den Bereitschaftsgraden, Niveaus, Interessen, Lernstilen und Intelligenzen der Schüler entsprechen. Diese Beurteilung ist ein kontinuierlicher diagnostischer Prozess. Zusätzlich zu Sprachförderkompetenz benötigen Lehrer auch Sprachdiagnosekompetenz (Ehl et al. 2018: 1236/ Reich et al. 2007: 7/ Geist 2014: 55).

Die Sprachdiagnoseverfahren können in vier Formen unterteilt werden, nämlich Schätzverfahren, die durch standardisierte Befragungen zur Selbst- und Fremdbewertung des Sprachniveaus des Schülers durchgeführt werden. Die zweite Form ist die Beobachtung, bei der das sprachliche Schülerhandeln in alltäglichen Kommunikationssituationen systematisch und differenziert beobachtet und beschrieben wird, ohne es zu

$$
\begin{aligned}
& \text {. ร)ภ. } \\
& \text { البحث التريوي }
\end{aligned}
$$




\section{المركن القومي للبحوث التهبويةوالنمية}

interpretieren. Die dritte Form ist die Profilanalyse, womit die freien Sprachproduktionen der Schüler durch Audiooder Videoaufnahmen erfasst werden. Danach werden sie hinsichtlich verschiedener sprachlicher Aspekte transkribiert und analysiert. Dies kann sehr zeitaufwendig sein, aber objektiver als die vorher genannten Verfahren. Die letzte Form der sprachdiagnostischen Verfahrensarten ist der Sprachtest, der in der Regel als differenziertes und komplexes Verfahren angesehen wird und sich auf einzelne sprachliche Aspekte beschränkt. Der Vorteil von Tests besteht darin, dass sie in hohem Maße vergleichbar sind und die einzelnen Sprachkenntnisse genauer untersuchen können (vgl. Kany/ Schöler 2010: 105; Ehlich 2005: 43f.).

In der vorliegenden Arbeit wurden verschiedene Sprachdiagnoseinstrumente verwendet, um die Effizienz des Einsatzes von der Sprachdiagnostik lernbegleitend beim Deutschlernen an der Oberschule zu untersuchen. 
Die Sprachdiagnostik zur Förderung des Deutschlernens

bei den Schülern an der Oberschule (Eine Fallstudie)

\section{Problemstellung}

Das Problem dieser Arbeit besteht in der Vernachlässigung der Sprachdiagnostik beim Deutschlernen und in der Beschränkung der Schülerevaluation beim Deutschlernen auf Tests. Diese Evaluation begleitet nicht den Lernprozess, ist für alle Schüler einheitlich und entspricht nicht den Bereitschaften, den Niveaus, den Interessen, den Lerntypen und den Intelligenzen aller Schüler.

Um dieses Problem zu behandeln, versucht die vorliegende Arbeit, auf die folgende Hauptfrage zu antworten:

Wie kann die Sprachdiagnostik als lernbegleitendes Evaluationsverfahren das Deutschlernen der Schüler an der Oberschule fördern?

Von dieser Hauptfrage verzweigen sich folgende Nebenfragen:

$$
\begin{aligned}
& . \Sigma Y . \\
& \text { البحث التريوي }
\end{aligned}
$$




$$
\text { المركن القومي للبحوث التبوبةوالتنمية }
$$

1. Welche Probleme haben die Schüler an der Oberschule beim Deutschlernen bezüglich der Sprachdiagnostik?

2. Was ist das auf der Sprachdiagnostik basierende Lernprogramm zur Förderung des Deutschlernens bei den Schülern an der Oberschule?

3. Inwieweit ist die Sprachdiagnostik wirksam für die Förderung des Deutschlernens bei den Schülern an der Oberschule?

\section{Zielsetzung der Arbeit}

Die Ziele der vorliegenden Arbeit sind:

a) Erkennen und Vorschlagen von Lösungen für Schülerprobleme beim Deutschlernen an der Oberschule.

b) Die Verwendung der Sprachdiagnostik als Evaluationsverfahren der individuellen sprachlichen Schülerleistung, damit die Förderungen gezielt eingesetzt werden. 
Die Sprachdiagnostik zur Förderung des Deutschlernens bei den Schülern an der Oberschule (Eine Fallstudie)

c) Die Untersuchung der Wirksamkeit der Sprachdiagnostik zur Förderung des Deutschlernens bei den Schülern an der Oberschule.

\section{Eingrenzung der Arbeit}

1. Kairo - Provinz, weil es in Kairo viele Schulen gibt, in denen Deutsch als zweite Fremdsprache gelernt wird.

2. Die zweite Klasse der Oberschule, damit die Kandidatin den Vortest durchführen kann, weil das Deutschlernen ab der ersten Klasse der Oberschule beginnt, und die Sprachstandserhebung Sprachwissen und -können erfordert.

3. Einige sprachdiagnostische Verfahren, die den Schülern an der Oberschule passen.

$$
\begin{aligned}
& \text {. SYT. } \\
& \text { البحث التريوي }
\end{aligned}
$$




$$
\text { المركن القومي للبحوث التبويةوالتنمية }
$$

\section{Hypothese der Arbeit}

Es entsteht einen Zusammenhang zwischen der Sprachdiagnostik und der Förderung des Deutschlernens bei den Schülern an der Oberschule.

\section{Terminologien der Arbeit}

Die Hauptterminologien dieser Arbeit sind die Sprachdiagnostik, die Sprachförderung und die Fallstudie. Im Folgenden werden sie ausführlich dargeboten;

\section{Sprachdiagnostik}

Der Begriff Sprachdiagnostik umfasst den gesamten Prozess der Sprachstandserhebung, der Sammlung sprachbiografischer Informationen, der Beurteilung und Interpretation der Daten bis hin zur Ableitung des individuellen Sprachförderbedarfs und der Förderziele (vgl. Lengyel 2012: 8).

Sprachdiagnostik bezeichnet ebenfalls die Analyse der vorhandenen Sprachkompetenzen und -kenntnisse anhand ausgewählter mündlicher oder schriftlicher .$\varepsilon r r$. 
Die Sprachdiagnostik zur Förderung des Deutschlernens bei den Schülern an der Oberschule (Eine Fallstudie)

Leistungsdaten und gilt als die Voraussetzung für eine möglichst genaue nachfolgende Förderung. Als Synonyme von Sprachdiagnostik werden oft die Begriffe Sprachstandsdiagnostik, Sprachdiagnose, Sprachstandsdiagnose, Sprachstandsmessung, Sprachstandserhebung und Sprachstandsbeobachtung benutzt. Sie beziehen sich alle auf eine evidenzbasierte Beurteilung der Sprachkompetenzen und -kenntnisse der Schüler (vgl. Jeuk/ Settinieri 2019: 3).

Sprachdiagnostik wird auch im Fachlexikon "Deutsch als Fremd- und Zweitsprache" als jeder Versuch definiert, das Sprachniveau des Schülers mit einer dem Ziel entsprechenden Methode aufzuzeichnen (vgl. Barkowski/ Krumm 2010: 309).

\section{Sprachförderung}

Das niedersächsische Kultusministerium (2011: 12) definiert die Sprachförderung als die begleitenden pädagogischen Aktivitäten zur Förderung und Unterstützung der Entwicklung von Sprachkenntnissen. 


$$
\text { المركن القومي للبحوث التبوبةوالتنمية }
$$

Sprachförderung bezeichnet auch alle Maßnahmen, die die Lehrer zugunsten der Unterstützung der Sprachentwicklung der Schüler einsetzen. Erfolg kann dabei nur erreicht werden, wenn die individuellen Lernbedürfnisse der Schüler in Betracht gezogen und darauf aufbauend unterstützt werden (vgl. Hellrung 2016: 82; Grießhaber/ Heilmann 2016: 6).

\section{Die Fallstudie}

Die meisten Definitionen von Fallstudien betonen die Einzigartigkeit des Falls, die Wichtigkeit des Kontexts, die Lieferbarkeit mehrerer Datenquellen oder Gesichtspunkte auf Beobachtungen und die Intensität der Analyse. Die Fallstudienmethode beinhaltet alle erforderlichen Verfahren zur Durchführung der Fallstudien. Das umfasst das Entwerfen einer Fallstudie, die Sammlung von Studiendaten, das Analysieren von Daten, das Vorstellen und Mitteilen von Ergebnissen (Duff 2018: 22; Yin 2012: $3)$. 
Die Sprachdiagnostik zur Förderung des Deutschlernens bei den Schülern an der Oberschule (Eine Fallstudie)

\section{Methodisches Vorgehen}

Um die Forschungsfragen $\mathrm{zu}$ beantworten, folgt diese Forschungsarbeit den folgenden Schritten:

\section{Bestimmung der Schülerprobleme an der Oberschule} beim Deutschlernen bezüglich der Sprachdiagnostik durch:

a. Sichtung der Fachliteratur $\mathrm{zu}$ den Themen Fremdsprachenlernprobleme, individuelle Förderung und individualisiertes Lernen im Fremdsprachenunterricht.

b. Umsetzung einer von der Kandidatin erstellten Schülercheckliste, um die Probleme und Erfahrungen der Schüler beim Deutschlernen kennenzulernen.

c. Umsetzung eines von der Kandidatin erstellten Lehrerfragebogens, um die Faktoren zu identifizieren, die zur individuellen Förderung der einzelnen Schüler während des Deutschlernens nach

$$
\text { البحثث التريوي }
$$




$$
\text { المركن القومي للبحوث التبوبةوالتنمية }
$$

der Meinung der Lehrer zu bestimmen und um zu erklären, ob diese Faktoren in der Unterrichtsrealität vorhanden sind.

d. Durchführung eines Interviews mit einigen Deutschlehrern, um deren Kenntnisse und Erfahrungen in Bezug auf die Leistungsbeurteilung anzuerkennen.

e. Aufbau eines diagnostischen Tests $\mathrm{zu}$ den Sprachfertigkeiten (Lesen, Schreiben, Hören und Sprechen) in kommunikativer Weise, der dem Sprachniveau A1 passt.

f. Erstellung von Bewertungskriterien der mündlichen und schriftlichen Schülerleistung im diagnostischen Test.

g. Durchführung des diagnostischen Tests.

h. Ergebnisanalyse des diagnostischen Tests, um die Stärken und Schwächen der einzelnen Schüler zu bestimmen. 
Die Sprachdiagnostik zur Förderung des Deutschlernens bei den Schülern an der Oberschule (Eine Fallstudie)

2. Realisierung des auf der Sprachdiagnostik basierenden Lernprogramms zur Förderung des Deutschlernens an der Oberschule durch:

a. Auswahl der Forschungsgruppe basierend auf den Testergebnissen. Die Forschungsgruppe bestand aus sechs Schülern. Drei von ihnen waren die erfolgreichsten und die anderen hatten die meisten Probleme bzw. Schwächen.

b. Aufbau eines entsprechenden Spezialprogramms für jeden individuellen Schüler von den vorher ausgewählten Schülern.

c. Erstellung eines Förderplans für jeden Schüler, der den eigenen Diagnosetest und die vorgeschlagenen Aufgaben und Aktivitäten im Laufe des Lernprogramms beinhaltet.

d. Dokumentieren der unterschiedlichen sprachlichen Leistungen des einzelnen Schülers und Einbeziehung dieser Leistungen in seinen persönlichen Förderplan.

$$
\begin{aligned}
& \text {. ¿ Y人. } \\
& \text { البحث التريوي }
\end{aligned}
$$




$$
\text { المركن القومي للبحوث التبوبةوالتنمية }
$$

\section{Beurteilung der Wirksamkeit der Sprachdiagnostik}

als Evaluationsverfahren für die Förderung der deutschsprachlichen Schülerleistung an der Oberschule durch:

a. Durchführung des diagnostischen Tests nochmal.

b. Analyse der Testergebnisse.

c. Erstellen einer Fallstudie (Case study) für jeden einzelnen Schüler.

d. Abfassung von Kommentaren zu den Ergebnissen.

e. Formulierung von Schlussfolgerungen und Empfehlungen.

\section{Forschungsergebnisse}

Wegen der wenigen Anzahl der Forschungsgruppe verwendete die Kandidatin den Wilcoxon-VorzeichenRang-Test als eine Art der nichtparametrischen Tests, um die Mittelwertdifferenzen der Forschungsgruppe in der ersten und letzten Durchführung des diagnostischen Tests (Vor- und Nachtest) zu ermitteln. Die Analyse der Ergebnisse ließ erkennen, dass es Differenzen bei einem 
Die Sprachdiagnostik zur Förderung des Deutschlernens

bei den Schülern an der Oberschule (Eine Fallstudie)

Signifikanzniveau von (0.05) zugunsten der zweiten Durchführung am Ende des Programms (Nachtest) gab. Diese Ergebnisse erklären, dass das auf der Sprachdiagnostik basierende Lernprogramm die Entwicklung des Sprachniveaus der Schüler effizient beeinflusste. Die Wirkungsgröße der Sprachdiagnostik auf die Förderung des Deutschlernens bei der Forschungsgruppe war sehr groß.

Die Kandidatin führte auch eine qualitative Analyse anhand der Fallstudien. Sie erstellte eine Fallstudie (Case study) für jeden einzelnen Schüler, die persönliche Informationen über den Schüler, Daten über seine Leistung in der ersten Durchführung des diagnostischen Tests (Vortest), über seine Leistung während des Programms, seine Probleme, seinen Förderplan, seine persönlichen Eigenschaften und über seine Leistung in der zweiten Durchführung des diagnostischen Tests (Nachtest) enthielt. Sie umfasste auch die Notizen der Kandidatin über den Schüler.

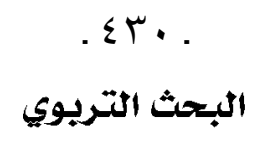




$$
\text { المركن القومي للبحوث التبوبةوالتنمية }
$$

\section{Schlussfolgerung und Konsequenzen für den DaF-Unterricht}

Die vorliegende Arbeit beschäftigte sich mit der Förderung des Deutschlernens bei den Schülern an der Oberschule durch den Einsatz der Sprachdiagnostik. Nach der Realisierung des auf der Sprachdiagnostik basierenden Lernprogramms kam die Kandidatin zu den folgenden Schlussfolgerungen;

$>$ Die Verwendung der Sprachdiagnostik schafft eine genaue Grundlage für das Lernen und ständige formative Beurteilung während des Lernens, um die einzelnen Schüler oder die Schülergruppe zielgerichtet zu fördern.

$>$ Der Schüler soll im Zentrum des Lernprozesses sein. So sollen seine Motivation, Stärken, Schwächen, Interessen, Lernvoraussetzungen und sein Vorwissen beachtet werden.

$>$ Beim Lernen bedeutet die Gerechtigkeit nicht die Gleichheit. Das bezieht sich darauf, dass der Lehrer 
Die Sprachdiagnostik zur Förderung des Deutschlernens

bei den Schülern an der Oberschule (Eine Fallstudie)

nicht mit allen Schülern gleich, sondern mit jedem Schüler je nach seinen Lernbedürfnissen individuell umgehen soll.

$>$ Der Unterricht soll möglichst individualisiert werden.

$>$ Die Diagnose, Förderung und die sprachdiagnostische Kompetenz sollen in die Ausund Fortbildung der Sprachlehrer einbezogen und trainiert werden.

$>$ Die Verwendung der begleitenden sprachdiagnostischen Verfahren führt zur Beseitigung der Angst vor Tests.

$>$ Durch die Verstärkung der Kooperation zwischen den Eltern und den Lehrern können die Probleme des Schülers gelöst werden und somit beeinträchtigen diese Probleme sein Lernen nicht.

$>$ Die Schaffung einer guten, sozialen und freundlichen Beziehung mit und unter den Schülern fördert das Lernen.

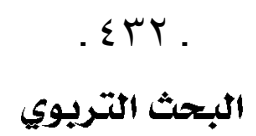




$$
\text { المركن القومي للبحوث التبوبةوالتنمية }
$$

$>$ Die Berücksichtigung der Schülermeinung über sein Lernen führt zur Verbesserung des Lernprozesses des betreffenden Schülers.

$>$ Die Berücksichtigung der kommunikativen Kompetenzen beim Trainieren im Unterricht sowie bei der Bewertung ist von grundlegender Bedeutung, denn die Sprache übt man nur kommunikativ in der Wirklichkeit.

> Die Profilanalyse gibt nur einen Überblick über die syntaktischen Fähigkeiten des Schülers, nicht jedoch über die Richtigkeit seiner sprachlichen Leistung.

$>$ Die Beobachtung ermöglicht eine Beschreibung der sprachlichen Leistung des Schülers aber nicht ihre Bewertung.

> Eine zutrauliche Leistungsbewertung erfordert eine geschickte Mischung der Bewertungsinstrumente.

$>$ Die Schule soll eine ständige individuelle Beratung darstellen. 
Die Sprachdiagnostik zur Förderung des Deutschlernens

bei den Schülern an der Oberschule (Eine Fallstudie)

$>$ Die Schule soll allen Schülern geeignete, herausfordernde, fördernde und aufregende Lernmöglichkeiten bieten.

$>$ Jedes Schulprogramm soll eine eigene Förderplanung enthalten. Sie soll allen Schülern ermöglichen, ihre Fähigkeiten so gut wie möglich zu entwickeln.

$>$ Lehrer sollen die Ergebnisse der Leistungsbewertungen als Basis für die Anpassung des Unterrichts an die Schülerbedürfnisse betrachten.

> Das Feedback der Lehrer soll den Schülern individuelle Rückmeldungen mit Förderangeboten bieten.

$>$ Lehrer sollen vielfältige Informationen über jeden individuellen Schüler sammeln.

> Lehrer sollen laufend die Fehler während des Schuljahres bearbeiten und interpretieren.

$>$ Die Leistungsbeurteilung kann die Kreativität und das Lernen behindern oder fördern. 


$$
\text { المركن القومي للبحوث التبويةوالتنمية }
$$

$>$ Der Einsatz von Sprachdiagnostik in der Unterrichtsrealität erfordert die Verringerung der Schüleranzahl in der Klasse, Verringerung der Unterrichtsstundenanzahl der Lehrer, damit die Lehrer ihre Schüler gut kennenlernen können und genügend Zeit für Datenerhebung $\mathrm{zu}$ den individuellen Schülern und für die Durchführung der diagnostischen Verfahren haben. Das erfordert auch die Zusammenarbeit der Lehrer, der Sozialspezialisten, der Schulleitung und der Familie. 
Die Sprachdiagnostik zur Förderung des Deutschlernens bei den Schülern an der Oberschule (Eine Fallstudie)

\section{Literaturverzeichnis}

1. Bakk. phil., Nicole Sylvia Huber (2014): Sprachstandsdiagnose - und dann? Exemplarische Sprachförderungsmaßnahmen nach einer Erhebung mit USB DaZ. Universität Wien.

2. Barkowski, Hans/ Krumm, Hans-Jürgen (Hrsg.) (2010): Fachlexikon Deutsch als Fremd-und Zweitsprache. Tübingen/ Basel: UTB.

3. Ditton, Hartmut (2018): Evaluation und Qualitätssicherung im Bildungsbereich. In Tippelt, Rudolf/ Schmidt-Hertha, Bernhard (Hrsg.): Handbuch Bildungsforschung. Wiesbaden: Springer VS. S. (757-777). 4. Duff, Patricia (2018): Case study research in applied linguistics. London/ New York: Routledge.

5. Ehl, Birgit/ Paul, Michèle/ Bruns, Gunnar/ Fleischhauer, Elisabeth/ Vock, Miriam/ Gronostaj, Anna/ Grosche, Michael (2018): Testgütekriterien der „Profilanalyse nach Grießhaber“. Evaluation eines Verfahrens zur Erfassung grammatischer Fähigkeiten von 


$$
\text { المركن القومي للبحوث التبويةوالتنمية }
$$

ein- und mehrsprachigen Grundschulkindern. Zeitschrift für Erziehungswissenschaft, 2018, 21.6: (1261-1281).

6. Ehlich, Konrad (2005): Sprachaneignung und deren Feststellung bei Kindern mit Migrationshintergrund: Was man weiß, was man braucht, was man erwarten kann. In: Ehlich, Konrad/Bredl, Ursula /Garme, Birgitta et al. (Hrsg.): Anforderungen an Verfahren der regelmäßigen Sprachstandsfeststellung als Grundlage für die frühe und individuelle Förderung von Kindern mit und ohne Migrationshintergrund. Bonn, Berlin: Bundesministerium für Bildung und Forschung. S. (11-77).

7. Ewringmann, Gisela/ Hruza-Mayer, Anne/ Huber, Franz/ Schätz, Josef (2004): Theoretisches Rahmenkonzept Evaluation Arbeitsgrundlage der Referate Q3 und Q4. Staatsinstitut für Schulqualität und Bildungsforschung Qualitätsagentur. München.

8. Geist, Barbara (2014): Sprachdiagnostische Kompetenz von Sprachförderkräften. Berlin/Boston: Walter de Gruyter GmbH.
9.
Gnahs,
Dieter/
Quilling, Eike
(2019): 
Die Sprachdiagnostik zur Förderung des Deutschlernens bei den Schülern an der Oberschule (Eine Fallstudie)

Qualitätsmanagement Konzepte und Praxiswissen für die Weiterbildung. Wiesbaden: Springer VS.

10. Gordon, Edmund W. / Rajagopalan, Kavitha (2016): The testing and learning revolution (The future of assessment in education), Palgrave Macmillan, USA.

11. Grießhaber,Wilhelm (Hrsg.)/ Heilmann, Beatrix (2016): Diagnostik \& Förderung-leicht gemacht. Stuttgart: Klett.

12. Hellrung, Uta (2016): Sprachentwicklung und Sprachförderung: beobachten - verstehen - handeln. Freiburg im Breisgau: Herder GmbH.

13. Jeuk, Stefan/ Settinieri, Julia (Hrsg.) (2019): Sprachdiagnostik Deutsch als Zweitsprache: ein Handbuch. Berlin/Boston: Walter de Gruyter GmbH \& Co KG.

14. Kany, Werner/ Schöler, Hermann (2010): Fokus: Sprachdiagnostik. Leitfaden zur Sprachstandsbestimmung im Kindergarten. Berlin: Cornelsen Scriptor.

15. Lengyel, Drorit (2012): Sprachstandsfeststellung bei mehrsprachigen Kindern im Elementarbereich. Deutsches

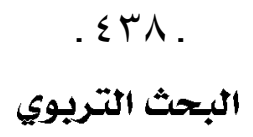




$$
\text { المركن القومي للبحوث التبويةوالتنمية }
$$

Jugendinstitut

e.V.

Weiterbildungsinitiative

Frühpädagogische Fachkräfte (WiFF).

16. Müller, Kirsten (2019): Fähigkeitsselbstkonzept, Beschulung und Unterrichtsqualität: Eine Untersuchung zum Zusammenhang des Fähigkeitsselbstkonzepts von Schüler* innen mit emotional-sozialem Förderbedarf, inklusiver und separater Beschulung und Unterrichtsqualität. Bad Heilbrunn : Julius Klinkhardt.

17. Niedersächsisches Kultusministerium (2011): Sprachbildung und Sprachförderung. Handlungsempfehlungen zum Orientierungsplan für Bildung und Erziehung im Elementarbereich niedersächsischer Tageseinrichtungen für Kinder. Hannover. URL: http://www. mk. niedersachsen. de/portal/live. php, 2011.

18. Reed, Daniel (2006): Diagnostic Assessment in Language Teaching and Learning, Clear News, VOLUME 10, ISSUE 2.

19. Reich, Hans (2006): Tests und Sprachstandsmessungen bei Schülern und Schülerinnen, .$\leqslant r q$. 
Die Sprachdiagnostik zur Förderung des Deutschlernens bei den Schülern an der Oberschule (Eine Fallstudie)

die Deutsch nicht als Muttersprache haben. In: Bredel, U. et al.: Didaktik der deutschen Sprache. Bd. 2. 2. Auflage. Paderborn, UTB- Schöningh. S. (914-923). 20. Reich, Hans H. / Roth, Hans-Joachim / Neumann, Ursula (Hrsg.) (2007): Sprachdiagnostik im Lernprozess. Verfahren zur Analyse von Sprachständen im Kontext von Zweisprachigkeit. FörMig Edition, Band 3. Münster.

21. Wiprächtiger- Geppert, Maja (2011): Sprachliche Leistungen beurteilen. In: Budde Monika/ Riegler, Susanne/ Wiprächtiger- Geppert, Maja (Hrsg.): Sprachdidaktik. Berlin: Akademie Verlag.

22. Yin, Robert K. (2012): Applications of case study research. USA: Sage. 